\title{
Alternatives to the Pre-operative Medical Management of Pheochromocytoma
}

Daniela Guevara, $M D^{1}$, Aryan Meknat, $M D^{1}$, Aida Taye Bellistri, $M D^{2}$, Alice C Levine, $M D^{3}$, William Inabnet III, $M D^{4}$ and Gustavo Fernández-Ranvier, $M D, P h D^{1^{*}}$
${ }^{1}$ Division of Metabolic, Endocrine and Minimally Invasive Surgery, Department of Surgery, Mount Sinai Hospital, Icahn School of Medicine at Mount Sinai, USA
${ }^{2}$ Department of Endocrine and General Surgery, Mount Sinai West \& St. Luke's Hospital, Icahn School of Medicine at Mount Sinai, USA
${ }^{3}$ Division of Endocrinology, Metabolism and Bone Diseases, Department of Medicine and Oncological Sciences, Icahn School of Medicine at Mount Sinai, USA
${ }^{4}$ Department of Surgery, University of Kentucky, KY, USA

\begin{abstract}
Aim of the study: During the resection of pheochromocytomas hemodynamic instability can occur. It is for this reason that pre-operative control of blood pressure and heart rate with non-selective alpha-blockers has been recommended. Other agents have emerged as alternatives to the preoperative management of symptoms of catecholamine excess. The purpose of this study is to analyze our experience with the use of the traditional non-selective alpha-blockers and to compare the pre-operative, intra-operative, and post-operative hemodynamics with the use of "other agents" including selective alpha-blockers, beta-blockers and calcium channel blockers.
\end{abstract}

Patients and methods: This study included 76 patients who underwent an adrenalectomy for pheochromocytoma between 1999 and 2014. The patients were grouped according to the type of pre-operative medication they had received. Patients in Group A ( $n=58)$ were treated with a non-selective alpha-blocker, Phenoxybenzamine \pm another agent. Patients in Group B ( $n=18)$ were treated solely with one or a combination of other agents: Doxazosin (selective alpha blocker), a beta-blocker, and/or a calcium-channel blocker. Heart rate and blood pressure were used as analogues for hemodynamic status and compared between the two groups.

Results: This investigation demonstrated significant differences; in the pre induction heart rate in group A with 81 bpm versus $73.2 \mathrm{bpm}$ in group B ( $p$ value 0.036 ); and in the post-operative systolic blood pressure, $124.2 \mathrm{mmHg}$ in group $A$ and $136 \mathrm{mmHg}$ in group $B$ ( $p$ value 0.016 ). Otherwise, there was no significant difference between both groups in controlling blood pressure and/or heart rate during the peri-operative period.

Conclusion: Our study showed that other agents such as selective alpha blockers, beta blockers and calcium channel blockers could be as effective as Phenoxybenzamine in controlling blood pressure and heart rate during the perioperative period.

Keywords

Pheochromocytoma, Hypertension, Doxazosin, Phenoxybenzamine

\section{Highlights}

- Hypertension is a common clinical finding in patients with pheochromocytoma.

- Pheochromocytoma is a rare neuroendocrine tumor in the adrenal gland.

- Preoperative treatment is recommended before surgery.

- Intraoperative instability can occur during the manipulation of the adrenal gland.
*Corresponding author: Gustavo Fernández-Ranvier, MD, $\mathrm{PhD}$, Division of Metabolic, Endocrine and Minimally Invasive Surgery, Department of Surgery, Mount Sinai Hospital, Icahn School of Medicine at Mount Sinai, 5 East 98th Street 15th floor New York, NY 10029, USA, Tel: 212-241-5339

Accepted: November 28, 2020

Published online: November 30, 2020

Citation: Guevara D, Meknat A, Bellistri AT, et al. (2020) Alternatives to the Pre-operative Medical Management of Pheochromocytoma. J Surgical Endocrinol 2(1):55-60

Copyright: ( 2020 Guevara D, et al. This is an open-access article distributed under the terms of the Creative Commons Attribution License, which permits unrestricted use, distribution, and reproduction in any medium, provided the original author and source are credited. 


\section{Introduction}

Pheochromocytomas (PCC) are rare neuroendocrine tumors originating from the adrenal medulla and/or extra-adrenal para-ganglionic tissues. These chromaffin tumors release an excess of active catecholamines; independent of the normal homeostatic negative feedback systems in place - though up to $20 \%$ can be biochemically silent [1]. Symptoms of catecholamine excess classically manifest as paroxysmal hypertension, episodic tachycardia, sweating, and/or headaches (i.e. adrenergic excess). PCC can vary in their presentation, from asymptomatic with episodic symptoms to continuous symptoms with intermittent adrenergic crises [1,2]. This all depends on the secretion profile of the tumor; the rate, the amount, and/or the predominant catecholamine (e.g. epinephrine versus norepinephrine) secreted $[1,3]$. Epinephrine (EPI) and Norepinephrine (NE) have different effects on adrenoceptor. EPI has more potent effect on beta2- adrenoceptor acting on receptors of the skeletal muscle vasculature causing vasodilation and hypotension. NE released locally from sympathetic nerve endings within the vasculature causes alpha1 adrenoceptor mediated vasoconstriction resulting in hypertension. Both catecholamines stimulate beta 1 adrenoceptor resulting in an increased heart rate. Patients with EPI-secreting tumors present episodic symptoms and signs including palpitations, light-headedness or syncope, anxiety and hyperglycemia than patients with tumors secreting predominantly NE, who more often have continuous symptoms and signs including hypertension, sweating and headache $[2,4]$. Diagnosis is made by a combination of biochemical and radiological tests to determine presence and location, respectively. Measuring plasma free metanephrines, which offers a diagnostic sensitivity of more than $97 \%$, establishes the diagnosis of PCC. Once the diagnosis of PCC has been confirmed its location can be found using CT scan or MRI. If the adrenal glands appear normal on either or both of these radiological modalities, MIBG scan (i.e. metaiodobenzylguanidine scan) may be considered but intra-adrenal pheochromocytomas are large with average size at time of diagnosis $4-5 \mathrm{~cm}$ so that CT or MRI should be sufficient for detection of tumors $[5,6]$. Incidence among the general population is approximately 0.8 per 100,000 person-years, and is estimated to be the source of high blood pressure in $0.2-0.6 \%$ of the hypertensive population [2].

Depending on the secretion profile of a PCC, the hemodynamic status of the patient can be highly variable. During an adrenalectomy, for a PCC, the handling and manipulation of the tumor may stimulate the release of large amounts of catecholamines into circulation, triggering significant hemodynamic changes [6]. This can be true even for patients with mild or a complete absence of signs and symptoms of catecholamines excess (i.e. asymptomatic and normotensive patients), or patients who may have PCC that are biochemically silent $[1,3,6]$. Before the advent of pre-operative medications to curtail the effects of catecholamine excess, hemodynamic instability encountered during surgery (e.g. hypertensive crisis, stroke, myocardial infarction, or cardiac and multi-organ failure) was responsible for significant morbidity and mortality [3].
Regardless of pre-operative hemodynamic status or associated symptomology, all patients undergoing adrenalectomy for PCC are setup for close intra-operative hemodynamic monitoring so that the consequences of intra-operative instability and catecholamine fluctuations are more swiftly controlled. This includes placement of invasive lines, delivery of anti-hypertensive and/or vasoactive drugs, and delivery of fluid boluses peri-operatively [5]. To prevent these life-threatening events (e.g. hypotension, shock, or hypertensive emergency), pre-operative medical management of blood pressure and heart rate is compulsory. This has historically been done with a non-selective alpha-blocker [6,7]. There is still debate as to which alpha-blockader is superior. To date, there have been studies comparing selective vs. non-selective alpha-blockers and alpha-blockers vs. calcium channel blockers on intra- and post-operative outcomes $[7,8]$. The aim of this study is to compare peri-operative hemodynamic status and peri-operative support requirements between patients who received non-selective alpha-blockers versus those who received selective alpha-blockers, beta-blockers, calcium channel blockers, or a combination of those listed.

\section{Patients and Methods}

Outcomes were analyzed from a prospectively maintained, Institutional Review Board (IRB) approved database. We retrospectively analyzed the medical records of 76 patients who underwent an adrenalectomy for a diagnosed PCC between 1999 and 2014. The tumors were diagnosed by correlating an elevation of plasma metanephrines and Normetanephrines with the presence of an adrenal mass. The diagnosis was confirmed on pathological analysis. Demographics such as age, gender, weight, and body mass index (BMI) were recorded. Plasma metanephrines/Normetanephrines levels, tumor size and location were also documented. Table 1 displays the patient demographics and tumor characteristics. Baseline blood pressure and heart rate, before pre-operative treatment, along with peri-operative blood pressures and heart rates were noted. Normotensive patients were also given alpha-blockade for pre-operative preparation.

The patients all underwent an operation after adequate preparation with non-selective alpha-blockade (Phenoxybenzamine), selective alpha-blockade (doxazosin), beta-blockade, calcium channel blockade, or with any of combination of the four - Table 2 illustrates the pre-operative drug therapy used for the groups. The adrenalectomies were performed either open or laparoscopically (via the transperitoneal or retroperitoneal route) under general anesthesia. The following variables were analyzed: surgical approach, duration of surgery, estimated blood loss, number of anti-hypertensives and/or vasoactive drugs required per patient, amount of intravenous (IV) fluids (crystalloids or colloids) infused, hemodynamic fluctuations (i.e. rise and/or fall of heart rate and blood pressure) before and after anesthesia induction, hemodynamic fluctuations during manipulation of the tumor, and hemodynamic fluctuations immediately after removal of the tumor. If or when hypotension occurred during the surgical procedure, it was managed with intravenous fluids and vasopressor drugs like norepinephrine, epinephrine, dobutamine, phenylephrine or vasopressin. 
Citation: Guevara D, Meknat A, Bellistri AT, et al. (2020) Alternatives to the Pre-operative Medical Management of Pheochromocytoma. J Surgical Endocrinol 2(1):55-60

Table 1: Demographics and tumor characteristics.

\begin{tabular}{|c|c|c|c|}
\hline & Group $A(n=58)$ & Group B $(n=18)$ & $P$ value \\
\hline \multicolumn{4}{|l|}{ Patient characteristic } \\
\hline Males & $20(34.5 \%)$ & $3(16.7 \%)$ & \\
\hline Females & $38(65.5 \%)$ & $15(83.3 \%)$ & \\
\hline Age (years) & $47.37(18-83)$ & $50(25-69)$ & \\
\hline $\mathrm{BMI}\left(\mathrm{kg} / \mathrm{m}^{2}\right)$ & $28.59 \pm 7.35$ & $30.82 \pm 5.98$ & \\
\hline \multicolumn{4}{|l|}{ Pheochromocytoma } \\
\hline Right adrenal & 26 & 7 & \\
\hline Left adrenal & 30 & 10 & \\
\hline Bilateral & 2 & 1 & \\
\hline Tumor size $(\mathrm{cm})$ & $5.36(1.4-10.5)$ & $5.25(11-2)$ & \\
\hline \multicolumn{4}{|l|}{ Catecholamines } \\
\hline Normetanephrines (nmol/l) & $12.47 \pm 14.62$ & $6.13 \pm 10.8$ & 0.094 \\
\hline Metanephrines (nmol/l) & $4.27 \pm 7.3$ & $3.19 \pm 5.54$ & 0.566 \\
\hline
\end{tabular}

Avg: Average; SD: Standard Deviation; BMI: Body Mass Index; Data are mean \pm SD: With the SD in parentheses.

Table 2: Pre-operative drug therapy.

\begin{tabular}{|l|l|l|}
\hline & Group A (n= 58) & Group B (n= 18) \\
\hline Phenoxybenzamine & $42(72.4 \%)$ & \\
\hline Phenoxybenzamine + Beta-blocker & $13(22.4 \%)$ & \\
\hline Phenoxybenzamine + Alpha-blocker & $1(1.7 \%)$ & $2(11.1 \%)$ \\
\hline Phenoxybenzamine + Calcium channel blocker & $2(3.4 \%)$ & $5(27.8 \%)$ \\
\hline Doxazosin & & $9(50 \%) 9$ \\
\hline Doxazosin + Beta-blocker & & $2(11.1 \%)$ \\
\hline Labetalol & & \\
\hline Calcium channel blocker+ Beta-blocker & & \\
\hline
\end{tabular}

Seventy six patients were divided into two groups: group $A(n=58)$ included patients who were treated in preoperative period with non-selective alpha-blockers (Phenoxybenzamine), and group $B(n=18)$ included patients treated with other medications; a selective alpha-blocker (Doxazosin only, $\mathrm{n}=2,11.1 \%)$, a selective alpha-blocker with a beta-blocker $(n=5,27.7 \%)$; a beta blocker alone $(n=9,50 \%)$, and a calcium channel blocker with a beta-blocker ( $n=2,11.1 \%)$. Most patients had unilateral pheochromocytomas except for 3 patients who had bilateral tumors $(n=2$ in group $A$ and $n=1$ in group $B$ ). Patients with an extra-adrenal pheochromocytoma were excluded from this study.

We assessed for significant differences in mean outcomes measured between the two groups by using paired t-tests to generate a two-tailed $p$-value. Statistical significance was defined as a $p$-value less than 0.05 . Calculations were performed with LaTeX.

\section{Results}

Pre-operatively, prior to receiving any medications the patients who ultimately were included in Group A had a mean blood pressure $(\mathrm{mmHg})$ of $142.3 / 81.9 \pm 29.9 / 16.3$ and mean heart rate $(\mathrm{bpm})$ of $78.5 \pm 15.8$ versus patients who ultimately were included in Group B who had a mean blood pressure of 130.2/77.1 $\pm 14.8 / 6.9$ and mean heart rate of $79.5 \pm 13.7$. After initiation of pre-operative medical management, for symptoms of catecholamine excess, patients in Group A had a mean blood pressure of 147.2/79 $\pm 15.4 / 8.7$ and mean heart rate of $81 \pm 13.7$ versus patients in Group B had a mean blood pressure of $144.5 / 74.5 \pm 19.7 / 13.6$ and mean heart rate of $73.2 \pm 12.6$. After induction of anesthesia, patients in Group $A$ had a mean blood pressure of 117.9/64.5 $\pm 16.8 / 10$ and mean heart rate of $74.4 \pm 12.1$ versus patients in Group B who had a mean blood pressure of 118.5/62.9 \pm 17.9/11.3 and mean heart rate of $73.1 \pm 15.8$. During the procedure (i.e. during handling and manipulation of the PCC) patients in Group A had a mean blood pressure of 122.3/68.1 \pm 14.4/7.4 and mean heart rate of $76.3 \pm 11.4$ versus patients in Group B had a mean blood pressure of 120.6/70.7 $\pm 11.3 / 10.4$ and mean heart rate of $75.6 \pm 12.8$. Within 24 hours of the adrenalectomy, patients in Group A showed a mean blood pressure of $124.2 / 72.1 \pm 17 / 11.3$ and mean heart rate of $74.1 \pm 12.4$ versus patients in Group B who had a mean blood pressure of $136 / 80.4 \pm 19.8 / 8.2$ and mean heart rate of $79.9 \pm 9.2$. Only the difference in mean heart rate measured after initiation of pre-operative medical management was found to be statistically significant $(p$-value $<0.05)$. Otherwise, there was no 
Citation: Guevara D, Meknat A, Bellistri AT, et al. (2020) Alternatives to the Pre-operative Medical Management of Pheochromocytoma. J Surgical Endocrinol 2(1):55-60

statistically significant difference found when comparing the blood pressure or heart rate of the two groups, measured at the different time intervals. Table 3 summarizes the results.

Sixty-five patients underwent laparoscopic adrenalectomy ( $n=49$ in group $A$ and $n=16$ in Group B), 5 patients ( $n=4$ in Group $A$ and $n=1$ in Group B) underwent an open adrenalectomy because tumor were malignant and was performed an extensive lymph nodes resection, three patients required radical nephrectomy and one tumor was around the vena cava. Six patients ( $n=5$ in group $A$ and $n=1$ in group B) underwent conversion from a laparoscopic into an open procedure because of the size of the tumor and its adherence

Table 3: Hemodynamic measures.

\begin{tabular}{|c|c|c|c|}
\hline Time of measurement & Group A ( $n=58)$ & Group B (n = 18) & $p$-value \\
\hline \multicolumn{4}{|c|}{ Pre-operative values before medical therapy } \\
\hline SAP & $142.3 \pm 29.9$ & $130.2 \pm 14.8$ & 0.103 \\
\hline DAP & $81.9 \pm 16.3$ & $77.1 \pm 6.9$ & 0.221 \\
\hline $\mathrm{HR}$ & $78.5 \pm 15.8$ & $79.5 \pm 13.7$ & 0.813 \\
\hline \multicolumn{4}{|c|}{ Pre-induction (general anesthesia) } \\
\hline SAP & $147.2 \pm 15.4$ & $144.5 \pm 19.7$ & 0.544 \\
\hline DAP & $79 \pm 8.7$ & $74.5 \pm 13.6$ & 0.101 \\
\hline HR & $81.0 \pm 13.7$ & $73.2 \pm 12.6$ & 0.036 \\
\hline \multicolumn{4}{|l|}{ Post-induction } \\
\hline SAP & $117.9 \pm 16.8$ & $118.5 \pm 17.4$ & 0.898 \\
\hline $\mathrm{HR}$ & $74.4 \pm 12.1$ & $73.1 \pm 15.8$ & 0.719 \\
\hline \multicolumn{4}{|c|}{ During procedure (i.e. during handling and manipulation of the PCC) } \\
\hline SAP & $122.3 \pm 14.4$ & $120.6 \pm 11.3$ & 0.659 \\
\hline DAP & $68.1 \pm 7.4$ & $70.7 \pm 10.4$ & 0.231 \\
\hline HR & $76.3 \pm 11.4$ & $75.6 \pm 12.8$ & 0.828 \\
\hline \multicolumn{4}{|c|}{ Peak post-operative values (within first week after surgery) } \\
\hline SAP & $124.2 \pm 17.0$ & $136 \pm 19.8$ & 0.016 \\
\hline DAP & $72.1 \pm 11.3$ & $80.4 \pm 8.2$ & 0.439 \\
\hline HR & $74.1 \pm 12.4$ & $79.9 \pm 9.2$ & 0.071 \\
\hline
\end{tabular}

*SAP: Systolic Arterial Pressure (mmHg); DAP: Diastolic Arterial Pressure (mmHg); HR: Heart Rate (beats per minute); Data are mean \pm SD: With the SD in parentheses

Table 4: Operative characteristics.

\begin{tabular}{|c|c|c|c|}
\hline & Group $A(n=58)$ & Group B $(n=18)$ & $P$ value \\
\hline \multicolumn{4}{|l|}{ ASA } \\
\hline 2 & 5 & 3 & \\
\hline 3 & 30 & 7 & \\
\hline 4 & 7 & 5 & \\
\hline LOS (days) & $3.8 \pm 2.98$ & $4.28 \pm 5.45$ & 0.632 \\
\hline Estimated blood loss (ml) & $195.13 \pm 234.36$ & $142.5 \pm 334.14$ & 0.457 \\
\hline Crystal solution infusion (ml) & $1961.11 \pm 1961.27$ & $1503.57 \pm 1266.81$ & 0.356 \\
\hline \multicolumn{4}{|l|}{ Procedure type } \\
\hline Laparoscopic & 49 & 16 & \\
\hline Open & 4 & 1 & \\
\hline Conversion & 5 & 1 & \\
\hline Operative time (min) & $166.07 \pm 80.84$ & $145.25 \pm 43.97$ & 0.301 \\
\hline
\end{tabular}

ASA: American Society of Anesthesiologists; ASA 2: Patient with a mild systemic disease; ASA 3: Patient with severe systemic disease; ASA 4: Patient with severe systemic disease that is a constant threat to life; Data are mean \pm SD: With the SD in parenthesis. 
to the kidney, vena cava and surrounding structures. Also the one of tumor infiltrated the adrenal vein and another patient required a nephrectomy. Group A required more intra-operative IV fluids but the difference was not statistically significant. Estimated blood loss, length of stay, operative time, volume of crystalloid and colloid solution infusion during the operation for Groups A and B are displayed in Table 4. Both groups required administration of exogenous electrolytes on multiple occasions, with no clinically or statistically significant difference. The use of intra-operative medications, anti-hypertensive and/or vasoactive drugs, was highly variable and a detailed account of their use is presented in Table 5. There was no statistically significant difference in the mean amount used intra-operatively, except for mean amount of Phentolamine (vasoactive drug) used on Group A (99.1 $\pm 96.28 \mathrm{mg}$ ) versus Group B (27.5 $\pm 30.72 \mathrm{mg})$.

\section{Discussion}

The optimal pre-operative alpha-receptor blockade plan, for the management of PCC, is still controversial [7]. Phenoxybenzamine has until recently been the mainstay therapy for the management of PCC, a non-selective alpha-receptor antagonist [8]. This medication is usually combined with a beta-receptor antagonist to suppress the reflexive tachycardia occurring as a result of elevated catecholamines interacting with beta-adrenergic cardiac receptors $[9,10]$. The pharmacologic effects persist long after the drug has been discontinued and patients can show clinical signs of persistent alpha-receptor blockade well into the postoperative period [1,8-11]. It is not uncommon for patients to develop persistent arterial hypotension and peripheral edema that will inevitably prolong the post-operative recovery phase [10]. Selective competitive alpha 1-receptor blockers, such as Doxazosin, have been advocated as an alternative to the phenoxybenzamine [10]. This selective alpha-blocker subtype has no effect on presynaptic alpha 2 or beta-receptors $[1,10]$. As doxazosin does not block the presynaptic alpha 2-receptors, which regulate norepinephrine release at adrenergic nerve endings, it is unnecessary to administer beta-receptor antagonists unless the patient has a predominantly epinephrine secreting tumor
$[1,2]$. An advantage of competitive selective alpha 1-receptor blockers is that, once the tumor has been removed and excess catecholamine release eliminated, alpha-receptor sensitivity quickly returns to normal function, leading to less post-operative hypotension [8]. Calcium channel blockers are another alternative agent for patients with very mild hypertension or severe hypotension under alpha-adrenergic receptor blockers $[8,10]$. These drugs reduce arterial pressure by inhibiting norepinephrine-mediated trans-membrane calcium influx in vascular smooth muscle cells. The use of a calcium channel blocker can also prevent the catecholamine mediated coronary artery spasm as well as control rhythm and heart rate [8]. It is common practice to only use beta-blockers after having started an alpha-blocker, unless specifically prescribed for a tachyarrhythmia. This is due to the fear that the patient will develop unopposed alpha-receptor overstimulation - with resultant hypertensive crisis [4]. However, 9 patients in the current study received preoperative therapy with beta-blockers alone and had no increase in morbidity or mortality. Our study showed that pre- and peri-operative management of patients with PCC can be done with alternative drugs, without compromising patient safety during the peri-operative period (i.e. pre-operatively, during induction, during handling/manipulation of the adrenal tissue, and post-operatively). There was no significant difference in blood pressure or heart rate during any time interval, except for the heart rate measured after initiation of pre-operative medical management. We hypothesize that the lower mean heart rate amongst patients in Group B was attributed to the alternative drugs being used (i.e. calcium channel blockers, beta-receptor blockers, and/or a combination of drugs) having a negative chronotropic effect on the heart.

After ligation of adrenal gland vessels and subsequent resection, the hypotension may be severe and it can be controlled by epinephrine, norepinephrine, neosynephrine, ephedrine, dopamine, and vasopressin - especially in patients receiving phenoxybenzamine $[8,10,12]$. This hypotension can be a consequence of the patient being on a long acting non-specific alpha-adrenergic blocking agent (i.e. phenoxybenzamine) and/or blood volume depletion (secondary to the

Table 5: Intra-operative drug therapy.

\begin{tabular}{|c|c|c|c|}
\hline & Group A $(n=58)$ & Group B $(n=18)$ & $P$ Value \\
\hline \multicolumn{4}{|l|}{ Anti-hypertensive drugs } \\
\hline Phentolamine (mg) & $99.1 \pm(96.28)$ & $27.5 \pm(30.72)$ & 0.03 \\
\hline Labetalol (mg) & $11.7 \pm(2.88)$ & $13.7 \pm(8.54)$ & 0.23 \\
\hline Esmolol (mg) & $175.4 \pm(215.59)$ & $294.8 \pm(384.71)$ & 0.12 \\
\hline \multicolumn{4}{|l|}{ Vasoactive drugs } \\
\hline Phenylephrine (mcg) & $388.2 \pm(323.65)$ & $586.7 \pm(821.08)$ & 0.19 \\
\hline Nitroglycerin (mcg) & $447.9 \pm(207.63)$ & $180 \pm(254.03)$ & 0.07 \\
\hline Nitroprusside (mcg) & $128.3 \pm(10.84)$ & $230 \pm(127.28)$ & 0.12 \\
\hline Norepinephrine (mcg) & $66.6 \pm(67.09)$ & $37.1 \pm N / A$ & N/A \\
\hline Vasopressin (Unit) & 0 & $4.4 \pm(3.43)$ & $\mathrm{N} / \mathrm{A}$ \\
\hline Epinephrine (mcg) & 0 & $1910 \pm N A$ & N/A \\
\hline
\end{tabular}

Data are mean $\pm S D$ : With the SD in parentheses. 
patient being on diuretics, from excess blood loss, or inadequate volume loading during the operation) [8]. If the clinical situation is dominated by hypotension, a purely alpha-adrenergic agonist is preferred (e.g. neosynephrine). If hypotension and bradycardia, both an alpha and beta-adrenergic agonist dominate, the clinical situation (e.g. epinephrine) should be used. Situations can arise where there is catecholamine resistant hypotension in which vasopressin is required for hemodynamic support. Neither patients in Group A nor B had episodes of post-operative hemodynamic instability.

\section{Conclusion}

This investigation has limitation including the use of inhomogeneous groups of patients, a small sample size and the retrospective analysis of the study. The results shown that other agents (including selective alpha blockers, beta blockers and calcium channel blockers)could be as effective as Phenoxybenzamine in controlling arterial blood pressure and heart rate during the pre- and peri-operative periods in patients with PCC. Alternatives to the traditionally used non-selective alpha-receptor blockers are also more cost-effective, meaning that more financially conservative options are available - without compromising efficacy and safety. Maintaining hemodynamic stability during the peri-operative period can be challenging for patients undergoing an adrenalectomy for PCC. Regardless of the medication or combination of medications used for pre-operative preparation for the surgery, there was no clinical or statistical difference in the measures used to maintain hemodynamic stability (i.e. volume of fluids administered, amount of vasoactive and/or anti-hypertensive delivered intra-operatively).

\section{Conflict of Interest}

The authors declare that they have no competing interest.

\section{Limitation}

Sample size small and inhomogeneous groups.

\section{Acknowledgement}

Supported by grant TL1TR0014341 from the National Center for Advancing Translational Sciences, National Institutes of Health and Mount Sinai Hospital, surgery department.

\section{References}

1. Sandi-Jo Galati, Meena Said, Rebekah Gospin, et al. (2015) The Mount Sinai clinical pathway for the management of pheochromocytoma. Endocrine Practice 21: 368-382.

2. PA van der Zee, A de Boer (2014) Pheochromocytoma: A review on preoperative treatment with phenoxybenzamine or doxazosin. The Netherlands Journal of Medicine 72: 190-201.

3. M Lafont, C Fagour, M Haissaguerre, et al. (2015) Per-operative hemodynamic instability in normotensive patients with incidentally discovered pheochromocytomas. J Clin Endocrinol Metab 100: 417-421.

4. Pacak K (2007) Preoperative management of the pheochromocytoma patient. J Clin Endocrinol Metab 92: 4069-4079.

5. Jacques WM Lenders, Karel Pacak, Thanh-Truc Huynh, et al. (2009) Low sensitivity of glucagon provocative testing for diagnosis of pheochromocytoma. J Clin Endocrinol Metab 95: 238245.

6. (2019) Pheochromocytoma (adrenaline-producing Adrenal Tumor). The American Association of Endocrine Surgery - Education Site.

7. Reese W Randle, Courtney J Balentine, Susan C Pitt, et al. (2017) Selective versus non-selective alpha-blockade prior to laparoscopic adrenalectomy for pheochromocytoma. Ann Surg Oncol 24: $244-250$.

8. Goldstein DP, Voigt MR, Ruan D (2017) Current preoperative preparation of pheochromocytoma/paraganglioma syndrome. Clin Surg 2: 1517.

9. Agrawal R, Mishra SK, Bhatia E, et al. (2014) Prospective study to compare peri-operative hemodynamic alterations following preparation for pheochromocytoma surgery by phenoxybenzamine or prazosin. World J Surg 38(3):716-723.

10. Domi R, Laho H (2012) Management of pheochromocytoma: Old ideas and new drugs. Niger J Clin Pract 15: 253-257.

11. Prys-Roberts C, Farndon JR (2002) Efficacy and safety of doxazosin for perioperative management of patients with pheochromocytoma. World J Surg 26: 1037-1042.

12. Harish Ramakrishna (2015) Pheochromocytoma resection: Current concepts in anesthetic management. J Anaesthesiol Clin Pharmacol 31: 317-323.

DOI: $10.36959 / 608 / 448$

Copyright: (c) 2020 Guevara D, et al. This is an open-access article distributed under the terms of the Creative Commons Attribution License, which permits unrestricted use, distribution, and reproduction in any medium, provided the original author and source are credited. 